\title{
1. Computing the Symbol
}

In this section, we compute the symbol of the scattering matrix - in particular given two magnetic fields which agree to some order, we compute the principal symbol of the difference of the associated scattering matrices in terms of the lead term of the difference of the magnetic fields. We use the techniques of [8], [5] and [4] to construct the Poisson operator and compute the symbols. We proceed explicitly where possible but occasionally fall back on results from [8] for brevity.

Let $P$ be the operator,

$$
P=\sum_{j=1}^{n}\left(i \frac{\partial}{\partial x_{j}}+A_{j}\right)^{2}+V=\Delta+i \sum_{j=1}^{n} A_{j} \frac{\partial}{\partial x_{j}}+q
$$

where $\left(A_{1}, \ldots, A_{n}\right)$ and $V$ are real-valued classical symbols of order -2 . The Poisson operator is then the map that maps $f \in C^{\infty}\left(S^{n-1}\right)$ to the smooth function $u$ such that $\left(P-\lambda^{2}\right) u=0$, and

$$
u=e^{i \lambda|x|}|x|^{-\frac{n-1}{2}} f(x /|x|)+e^{-i \lambda|x|}|x|^{-\frac{n-1}{2}} g(x /|x|)+O\left(|x|^{-\frac{n+1}{2}}\right) .
$$

The kernel of the Poisson operator will be a smooth function on $S^{n-1} \times \mathbb{R}^{n}$ with singular asymptotics. The scattering matrix is the map on $C^{\infty}\left(S^{n-1}\right)$,

$$
S(\lambda): f \mapsto g
$$

Our first result in this section is

Proposition 1.1. With $\left(A_{1}, \ldots, A_{n}\right)$ and $q$ as above, we have that $a^{*} S(\lambda)$ is a zeroth order classical pseudo-differential operator, where $a(\omega)=-\omega$.

We remark that saying that $a^{*} S(\lambda)$ is a zeroth order classical pseudodifferential operator is equivalent to saying that is a classical Fourier integral operator of order 0 associated with geodesic flow at time $\pi$. This proposition is clear from any of [8], 四] and [9] by just observing that the arguments are not changed by adding a first order short range self-adjoint perturbation, we therefore only present the parts of the proof which relate to the proof of our main result:

Theorem 1.1. Let $\left(A_{1}, \ldots, A_{n}\right)$ and $\left(A_{1}^{\prime}, \ldots, A_{n}^{\prime}\right)$ be real-valued classical symbols of order -2 with $S(\lambda)$ and $S^{\prime}(\lambda)$ the associated scattering matrices. If $A_{j}-A_{j}^{\prime}$ is of order $-k$ with $k \geq 2$ then $S(\lambda)-S^{\prime}(\lambda)$ is of order $1-k$. If $\sum_{j=1}^{n}\left(A_{j}-A_{j}^{\prime}\right) d x_{j}=B$ with lead term $B^{(k)}$, and $B^{(k)}$ is aradial then the principal symbol of $S(\lambda)-S^{\prime}(\lambda)$ determines and is determined by

$$
\int_{0}^{\pi}\left\langle B^{(k)}(\gamma(s)), \frac{d \gamma}{d s}(s)\right\rangle(\sin s)^{k-1} d s
$$


for all geodesics $\gamma$, where we regard $B^{(k)}=\sum B_{j}^{(k)} d x_{j}$ as a one form canonically pairing with the vector $\frac{d \gamma}{d s}(s)$.

We say a one-form is aradial if its pairing with the radial vector field, $x \frac{\partial}{\partial x}$, is zero.

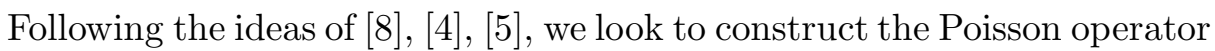
for the problem as a sum of oscillatory integrals and then use this to read off the properties of the scattering matrix. In particular, we attempt to construct the Poisson operator as

$$
e^{i \lambda x \cdot \omega}(1+b(x, \omega))
$$

with $b$ a classical symbol of order -1 in $x$ and smooth in $\omega$. We will see that this ansatz works away from the set $\omega=-x /|x|$. Applying $P-\lambda^{2}$, we obtain

$$
e^{i \lambda x \cdot \omega}\left(-2 i \lambda \omega \cdot \frac{\partial b}{\partial x}+\Delta b-\lambda \sum_{j=1}^{n} \omega_{j} A_{j}(1+b)+i \sum_{j=1}^{n} A_{j} \frac{\partial b}{\partial x_{j}}+q+q b\right) .
$$

That this can be solved smoothly to infinite order in a neighbourhood of $x /|x|=\omega$ is just a repetition of the argument in the proof of Proposition 18 of [8].

We let $b$ have asymptotic expansion $\sum_{j=1}^{\infty} b_{-j}$, let $A_{l}$ have expansion $\sum_{j=2}^{\infty} A_{l}^{(-j)}$ and $q$ have expansion $\sum_{j=2}^{\infty} q_{-j}$. In order to continue $b$ smoothly we observe that the lead term is

$$
-2 i \lambda \omega \cdot \frac{\partial b}{\partial x}-\lambda \sum_{j=1}^{n} \omega_{j} A_{j}^{(-2)}+q_{-2} .
$$

We want this to be zero.

Note as these are homogeneous functions this is really an equation on the sphere. We therefore take coordinates $(r, s, \theta)$ where $r=|x|, s$ is the geodesic distance of $x /|x|$ from $\omega$ and $\theta$ is the angular coordinate about $\omega$. Note the coordinate system depends on $\omega$ but we shall suppress $\omega$ in our notation most of the time.

Now with out loss of generality, we can take $\omega$ to be the north pole. Then

$$
\omega . \partial_{x}=\partial_{x_{n}} .
$$

Now $\cos (s)=\frac{x_{n}}{|x|}, r=|x|$. The $\theta$ coordinate will be purely parametric. We have

$$
\frac{\partial s}{\partial x_{n}}=\frac{-1}{\sqrt{1-\frac{x_{n}^{2}}{|x|^{2}}}}\left(\frac{1}{|x|}-\frac{x_{n}^{2}}{|x|^{3}}\right)=-r^{-1} \sin (s),
$$


and

$$
\frac{\partial r}{\partial x_{n}}=\cos (s)
$$

So applying $\omega . \partial_{x}$ to $b_{-j}(s, \theta ; \omega) r^{-j}$, we obtain

$$
r^{-j-1}\left[-\sin (s) \frac{\partial b_{-j}}{\partial s}-j \cos (s)\right] b_{-j}
$$

Let $W_{-2}=-\lambda \sum_{j=1}^{n} \omega_{j} A_{j}^{(-2)}-q_{-2}$. So for $j=1$, we have taking $r=1$

$$
2 i \lambda\left(\sin (s) \partial_{s}+\cos (s)\right) a_{-1}=W_{-2},
$$

which is equivalent to

$$
2 i \lambda \partial_{s}\left(\sin (s) a_{-1}\right)=W_{-2} .
$$

We want $b$ to be smooth at $s=0$, so

$$
\sin (s) b_{-1}=\frac{1}{2 i \lambda} \int_{0}^{s} W_{-2} d s^{\prime}
$$

which implies that

$$
a_{-1}(s, \theta ; \omega)=\frac{i}{2 \lambda \sin (s)} \int_{0}^{s} W_{-2}\left(s^{\prime}, \theta ; \omega\right) d s^{\prime} .
$$

This will be singular as $s \rightarrow \pi-$, but let's ignore that for now. Now suppose we have chosen the first $j$ terms so we have an error $d \in S_{c l}^{-j-1}$ with lead term $d_{-j-1}(s, \theta ; \omega) r^{-j-1}$. We then want to solve the transport equation,

$$
-2 i \lambda \omega \cdot \partial_{z}\left(a_{j} r^{-j}\right)+d_{-j-1} r^{-j-1}=0
$$

as above we get

$$
2 i \lambda\left[\sin (s) \partial_{s}+j \cos (s)\right] a_{j}+d_{-j-1}=0 .
$$

We solve this to obtain,

$$
a_{j}(s, \theta, \omega)=\frac{i}{2 \lambda(\sin (s))^{j}} \int_{0}^{s}\left(\sin \left(s^{\prime}\right)\right)^{j-1} d_{-j-1}\left(s^{\prime}, \theta ; \omega\right) d s^{\prime} .
$$

So away from $s=\pi$, we can achieve an error in $S^{-\infty}$ by applying Borel's lemma. ie away from the antipodal point. Note that we have a focussing of the geodesics and as well as the fact the solutions blow-up we also have that they will have different values according to the angle. In particular provided $d_{-j-1}$ does not grow faster than $(\pi-s)^{1-j}$ we have that $a_{j}$ grows as $(\pi-s)^{-j}$.

Before introducing a second ansatz to cope with the antipodal point, we compare the Poisson operators associated to two different magnetic potentials. Suppose $\left(A_{1}, \ldots, A_{n}\right)$ and $\left(A_{1}^{\prime}, \ldots, A_{n}^{\prime}\right)$ are both classical symbols of order -2 and the difference is $\left(B_{1}, \ldots, B_{n}\right)$ which is a classical symbol of 
order $-k$, with lead term $\left(B_{1}^{(-k)}, \ldots, B_{n}^{(-k)}\right)$. The first $k-2$ forcing terms above are then unchanged and the forcing terms at level $k-1$ will differ by $W_{-k}=-\lambda \sum_{j=1}^{n} \omega_{j} B_{j}^{(-k)}$. (Note that the change in the zeroth order term will be lower order.)

Thus the lead term of the difference of the Poisson operators will be

$$
\frac{i r^{1-k}}{2 \lambda(\sin s)^{k-1}} \int_{0}^{s} W_{-k}\left(s^{\prime}, \theta ; \omega\right)\left(\sin s^{\prime}\right)^{k-2} d s^{\prime} .
$$

This is the important result in our construction as we will see that the lead term of this as $s \rightarrow \pi-$ is essentially the principal symbol of the difference of the scattering matrices. We therefore want an invariant interpretation of

$$
\int_{0}^{\pi}(\sin s)^{k-2} W_{-k}\left(s, \theta^{\prime} ; \omega\right) d s .
$$

If we take $\omega$ to be the north pole and rotate so that $\theta^{\prime}=(1,0, \ldots, 0)$, the computation lies entirely in the $\left(x_{1}, x_{n}\right)$ plane and $W_{k}(s)$ equals $-\lambda B_{n}^{(-k)}(s)$. Now if we assume $B$ is aradial then an elementary computation shows that,

$$
-\left\langle B^{(-k)}(\gamma(s)), \frac{d \gamma}{d s}(s)\right\rangle \sin s=B_{n}^{(-k)}
$$

in this case. So by rotational invariance we deduce that in general the lead term of the difference of the Poisson operators is

$$
\frac{i r^{1-k}}{2(\sin s)^{k-1}} \int_{0}^{s}\left\langle B^{(-k)}\left(\gamma\left(s^{\prime}\right)\right), \frac{d \gamma}{d s^{\prime}}\left(s^{\prime}\right)\right\rangle\left(\sin s^{\prime}\right)^{k-1} d s^{\prime}
$$

and that the lead singularity as $s \rightarrow \pi-$ is

$$
\frac{i r^{1-k}}{2(\pi-s)^{k-1}} \int_{0}^{\pi}\left\langle B^{(-k)}\left(\gamma\left(s^{\prime}\right)\right), \frac{d \gamma}{d s^{\prime}}\left(s^{\prime}\right)\right\rangle\left(\sin s^{\prime}\right)^{k-1} d s^{\prime} .
$$

The remainder of the construction of the Poisson operator and the computation of the symbol is now just a repetition of the arguments in [8] or [四. We sketch these for completeness.

Taking $\omega$ to be the north pole, close to the south pole we look for the Poisson operator in the form, So close to the south pole, we look for the Poisson operator in the form,

$$
\int_{0}^{\infty} \int\left(\frac{1}{S|x|}\right)^{\gamma} S^{\alpha}\left(\frac{1}{S|x|}\right)^{\gamma} S^{\alpha} e^{i\left(S x^{\prime} \cdot \mu-\sqrt{1+S^{2}}|x|\right)} a\left(\frac{1}{S|x|}, S, \mu\right) d S d \mu,
$$

with $a(t, S, \mu)$ a smooth function compactly supported on $[0, \epsilon) \times[0, \epsilon) \times S^{n-2}$ and $\alpha=\frac{n-3}{2}, \gamma=-\frac{n-1}{2}$. We assume that $\omega$ has been rotated to the north pole. Note that for $|x|$ in a compact set the integral is supported on a 
compact set and so we have no problems with convergence - in particular the integral yields a smooth function.

In the lower hemi-sphere, away from the south pole, this ansatz is equivalent to the original one - this follows from an application of stationary phase (see [畉). However the lead term in $|x|$ at order $-k$ is now allowed to be singular of order $-k$ as $s \rightarrow \pi-$ (which corresponds to $S=0+$ ) and there is no constraint on the values for different angles matching. This allows the transport equations to be solved right up to the antipodal point and to all orders. The error is then removed by applying the resolvent which yields a term of the form $e^{-i \lambda|x|}|x|^{-\frac{n-1}{2}} h(x)$ with $h$ a classical zeroth order symbol - this term will not affect the singularities in the distributional asymptotics of the Poisson operator.

We recall Proposition 3.4 of [4], which is a special case of Proposition 16 of [8].

Proposition 1.2. If $u(x, \omega)$ is of the form 1.9 then $e^{i \lambda|x|} \int u(|x| \theta, \omega) f(\theta, \omega) d \theta d \omega$ is a smooth symbolic function in $|x|$ of order $-1-\alpha$ and its lead coefficient is $|x|^{-\alpha-1}\langle K, f\rangle$ where $K$ is the pull-back of the Schwartz kernel of a pseudodifferential operator of order $\alpha-\gamma-(n-2)$ by the map $\theta \mapsto-\theta$. The principal symbol of $K$ determines and is determined by the lead term of the symbol, $a(t, S, \mu)$, of $u$ as $S \rightarrow 0+$.

The fact that the scattering matrix is the pull-back of a pseudo-differential operator is now immmediate. To deduce Theorem 1.1, we observe that from our computations above we have that the difference of the second ansatzs for the two Poisson operators will be of the same form but with $\alpha$ increased by $k-1$ and the lead term of the symbol as $S \rightarrow 0+$ will be a constant multiple of

$$
S^{n-k-1} \int_{0}^{\pi}\left\langle B^{(-k)}\left(\gamma\left(s^{\prime}\right)\right), \frac{d \gamma}{d s^{\prime}}\left(s^{\prime}\right)\right\rangle\left(\sin s^{\prime}\right)^{k-1} d s^{\prime} .
$$

So the theorem then follows from Proposition 1.2.

\section{REFERENCES}

[1] G. Eskin, J. Ralston, Inverse Scattering Problem for the Schrodinger Equation with Magnetic Potential at a Fixed Energy, Commun. Math. Phys. 173, 199-224 (1995)

[2] S. Helgasson, Groups and Geometric Analysis, Academic Press 1984.

[3] M.S. Joshi, Recovering Asymptotics of Coulomb-like Potentials, to appear in S.I.A.M. Journal of Mathematical Analysis

[4] M.S. Joshi, Explicitly Recovering Asymptotics of Short Range Potentials, preprint

[5] M.S. Joshi, A. Sá Barreto, Recovering Asymptotics of Short Range Potentials, Comm. Math. Phys. 193, 197-208 (1998)

[6] M.S Joshi, A. Sá Barreto, Recovering Asymptotics of Metrics from Fixed Energy Scattering Data, to appear in Invent. Math. 
[7] R.B. Melrose, Spectral and Scattering Theory for the Laplacian on Asymptotically Euclidean spaces (M. Ikawa, ed), Marcel Dekker, 1994.

[8] R.B. Melrose, M. Zworski, Scattering Metrics and Geodesic Flow at Infinity, Invent. Math. 124, 389-436 (1996).

[9] A. Vasy, Geometric Scattering Theory for Long-Range Potentials and Metrics, I.M.R.N. 1998 no 6, 285-315

Department of Pure Mathematics and Mathematical Statistics, University of Cambridge, 16 Mill Lane, Cambridge CB2 1SB, England, U.K.

E-mail address: joshi@dpmms.cam.ac.uk 